\title{
SHOCK COMPRESSION AND RECOVERY OF MICROORGANISM- LOADED BROTHS AND AN EMULSION
}

\author{
P. J. Hazell ${ }^{1}$, C. Beveridge ${ }^{2,3}$, K. Groves ${ }^{2}$, C. Stennett ${ }^{1}$ \\ ${ }^{1}$ Cranfield Defence and Security, Cranfield University, Shrivenham, Swindon, SN6 8LA \\ ${ }^{2}$ Leatherhead Foods International, Randalls Road, Leatherhead, Surrey, KT22 7RY \\ ${ }^{3}$ Present address: CBC, 49 Adkin Way, Wantage, Oxfordshire, OX12 $9 H N$
}

\begin{abstract}
The microorganisms Escherichia coli, Enterococcus faecalis and Zygosaccharomyces bailii and an oil-based emulsion, have been subjected to shock compression using the flyer-plate technique to initial pressures of $0.8 \mathrm{GPa}$ (in the suspension). In each experiment, a stainless steel capsule was used to contain the broths and allow for recovery without contamination. Where cavitation was mostly suppressed by virtue of simultaneous shock and dynamic compression, no kill was observed. By introducing an air gap behind the suspension, limited kill was measured in the yeast. Results also suggest that stable emulsification occurs in coarse oil-based emulsions that are subjected to shock.
\end{abstract}

Keywords: Shock compression; microorganisms, yeast, capsule design.

PACS: $62.50 . \mathrm{Ef}$, 47.40.Nm, 47.57.Bc, 87.18-h

\section{INTRODUCTION}

Various attempts have been tried in the past to kill microorganisms via a process of inducing shock waves in a medium. On one hand, using weak underwater shock waves has been suggested as a method for food preservation [1]. Whereas on the other hand, researchers have focused their attention on the response of microorganisms to strong shock waves to elucidate possible mechanisms for Panspermia (i.e., microorganisms are distributed throughout the Universe and spread naturally to seed life) [2-4]. Loske et al. [1] reported a kill of microorganisms via a process of electro-discharge within a vat of water. Here they reported that multiple shock waves of c.a. $44 \mathrm{MPa}$ resulted in mortality of $E$. Coli with increasing death rates as the number of applied shock waves increased. Zuckerman et al. [5] used similar techniques to generate pressures of c.a. $100 \mathrm{MPa}$ resulting in inactivation in just 5 pulses. In this work we report results where we interrogate the mortality of microorganisms that have been subjected to shock compression. Using the flyer- plate technique we subject the samples to pressures that are much higher than those seen using electrodischarge techniques. Further, an oil-based emulsion has also been subjected to similar shock loading resulting in significant breakdown of the oil particles.

\section{EXPERIMENTAL PROCEDURE}

All the experiments were conducted using a 50-mm single-stage light gas-gun [6]. 10-mm thick copper flyer plates were accelerated to velocities of between $345 \mathrm{~m} / \mathrm{s}$ and $450 \mathrm{~m} / \mathrm{s}$ and were used to dynamically load a stainless steel capsule containing the suspension of interest. The measured shock magnitudes in the stainless steel cover plates that sealed the capsules, were c.a. 6.4 $\mathrm{GPa}$ and 8.6 GPa, corresponding to velocities of $345 \mathrm{~m} / \mathrm{s}$ and $450 \mathrm{~m} / \mathrm{s}$. The velocities of the projectile were measured by using a sequential pin shorting system to an accuracy of $0.5 \%$.

Two capsule designs were used to house the microorganisms and emulsion (see Fig 1). In the first experiments, the cavity within the capsule 


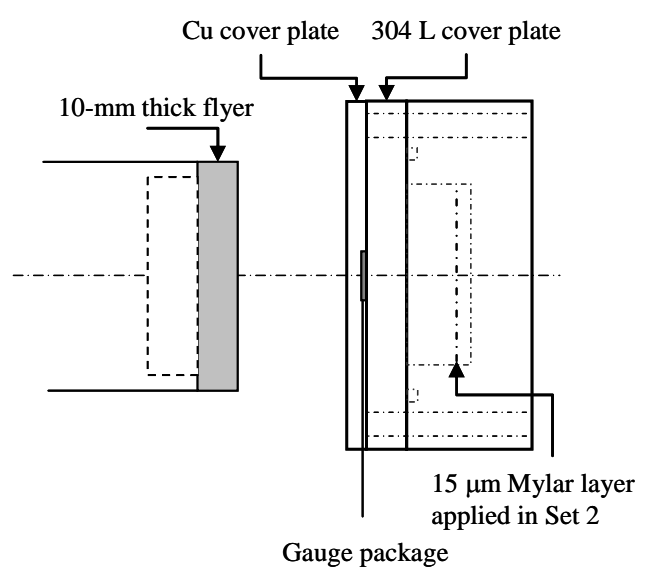

Figure 1. Experimental setup showing the arrival of a 10-mm thick Cu flyer.

was completely filled then sealed, taking care to minimize the presence of air.

In the second set of experiments, a $15 \mu \mathrm{m}$ Mylar layer was introduced to separate the fluid from the steel by c.a. $3 \mathrm{~mm}$ thereby providing an air gap. Six experiments were conducted in total; hydrocode (AUTODYN) simulations were also carried out to assess the dynamic deformation of the cover plate and the pressure history within the fluid layers.

The microorganisms used for this experiment were Zygosaccharomyces bailii DSM 70492 and a cocktail of Escherichia coli NCTC 9001 and Enterococcus faecalis, culture ATCC 19433. Z. bailii is a common spoilage yeast in the food industry chiefly affecting fruit juices; $E$. coli and $E$. faecalis are relatively harmless members of a healthy gut flora.

The Z. bailii experimental culture was prepared by incubating in malt extract broth (prepared from Oxoid powder) at $25^{\circ} \mathrm{C}$ for 3 days to provide a $10^{8}$ colony forming units/ml (cfu/ml). A cocktail of $E$. coli NCTC and E. faecalis culture ATCC 19433 9001 was incubated in nutrient broth (prepared from Oxoid powder) at $37^{\circ} \mathrm{C}$ for 1 day to achieve the $10^{8} \mathrm{cfu} / \mathrm{ml}$. These cultures were then individually subjected to the shock loading.

After subjecting the capsules to the shockloading they were recovered and opened using aseptic techniques. A $1 \mathrm{ml}$ sample was withdrawn and serially diluted into 8 by $9 \mathrm{ml}$ of half-strength ringer's solution. $0.1 \mathrm{ml}$ of each of the dilutions was then plated onto nutrient agar for incubation and subsequent numerical evaluation.

\section{RESULTS AND DISCUSSION}

\section{Wave propagation}

Hydrocode simulations of each capsule design showed the response of the system to impact. Fig 2 shows one such example of a 10-mm thick copper flyer plate striking the capsule at c.a. $450 \mathrm{~m} / \mathrm{s}$. A shock magnitude of c.a. $8.5 \mathrm{GPa}$ was measured at the gauge location shown in Fig 1. This lasted for approximately $3.2 \mu$ s before the arrival of the release wave. After the flyer-plate struck the copper cover plate, the stainless steel plate was extruded forward. Simultaneously the shock passed into the suspension and subsequently rung-up resulting in peak pressures of $1.2 \mathrm{GPa}$ in the fluid layer. In doing so, a divergent wave front emerged by virtue of the fact that the stainless steel cover plate was extruding in a hemispherical fashion (see Fig 2). The simultaneous shock and dynamic compression of the fluid extended the time over which fluid was compressed and thereby mostly suppressed the formation of a negative phase that would otherwise lead to cavitation.

In the second set of experiments, an airgap was introduced behind the fluid layer by using a $15-\mu \mathrm{m}$

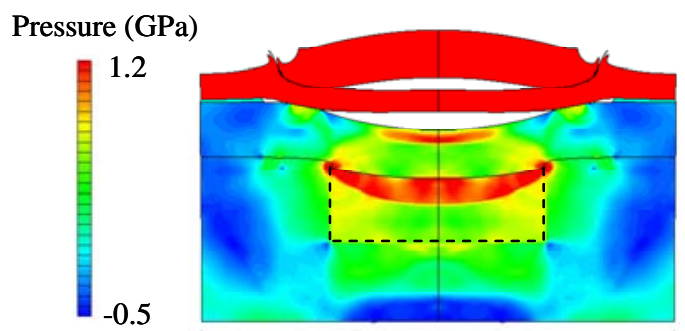

(a)

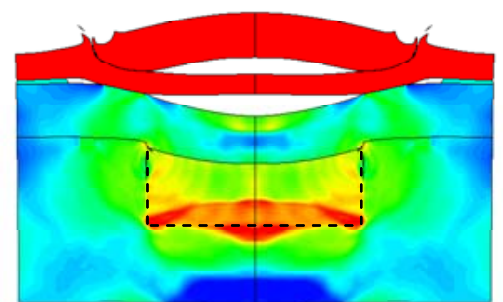

$+5 \mu \mathrm{s}$

Figure 2. ANSYS ${ }^{\circledR}$ AUTODYN simulation showing the deformation of the cover plate and the pressure in the water. 
thick layer of Mylar® film. This resulted in the the formation of a negative phase with the fluid layer being subjected to "stretching" [7]; this was assumed to lead to cavitation.

\section{Effect on the microorganisms}

Analysis of the shocked specimens showed that the compression pulse had little effect on the bacterial samples when cavitation was mostly suppressed; no reduction in numbers was observed when compared to the control (unshocked) specimen for both the bacteria and the yeast samples. This is somewhat surprising given the high compression that was achieved using this technique and given that other researchers have noted bacterial kill with much lower pressures [1,5]. This suggests that the mechanisms of kill were not dependent on solely the magnitude of the pressure but rather other factors such as the degree of repetition and exposure to UV light.

However, when the air-gap was introduced in the cavity limited kill was observed with 1-log reduction in the yeast sample only. Again, no measureable reduction was seen in the bacterial samples. Furthermore, it was noted that, with the yeast sample, clumping of the microorganisms was observed c.a. 30 minutes after the experiment (see Fig 3). The reason for this was unclear however it has previously been reported that certain microorganisms undergo aggregation during stress and therefore the observed behavior maybe a survival mechanism $[8,9]$.

The reason for the high survival rates of the microorganisms is, perhaps, surprising given the

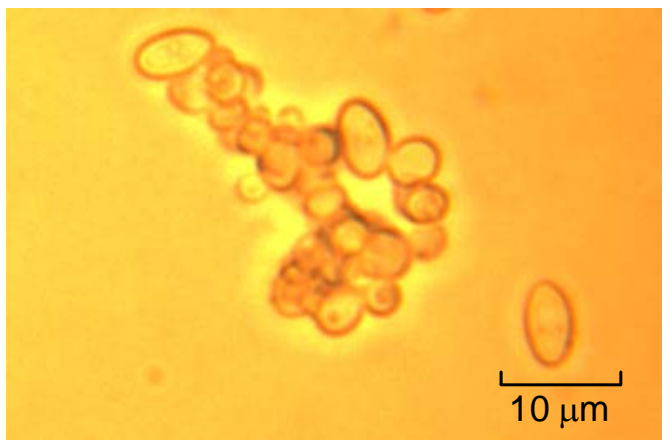

Figure 3. Aggregation of the yeast cells after shock compression. expected violence of the cavitation in the second set of experiments. These results confirm the hardy nature of certain micro-organisms that have been subjected to shock loading as noted by Burchell et al. [2]. The reason for high survival rates when subjected to shock loading for the cells contained in a suspension demands explanation and is probably due to the materials and structure of the cells. It should be pointed out that the cell make-up consists of materials that are of similar shock impedance to the fluid medium that contains them. Consequently, to kill these organisms it is probable that higher shocks are required to induce delamination of the cell structure.

\section{Effect on the emulsion}

Perhaps more enlightening are the effects that the shock wave had on the emulsification of the oil-based suspensions. The coarse emulsions prior to the passage of the shock wave possessed a volume mean particle size of $13.6 \mu \mathrm{m}$ with a distribution (by \% volume) of particle sizes as follows: $10 \% \leq 1.2 \mu \mathrm{m}, 50 \% \leq 13.0 \mu \mathrm{m}$ and $90 \% \leq$ $25.1 \mu \mathrm{m}$. After the passage of the shockwave, the droplet size distribution changed, producing smaller droplets with a volume mean particle size of $8.2 \mu \mathrm{m}$ and a distribution of: $10 \% \leq 1.2 \mu \mathrm{m}, 50 \%$ $\leq 7.3 \mu \mathrm{m}$ and $90 \% \leq 16.7 \mu \mathrm{m}$.

For the second experimental setup where cavitation was promoted, significant emulsification occurred as shown in Fig 4. After the passage of the shockwave, the droplet size distribution changed, producing smaller droplets with a volume mean particle size of $7.8 \mu \mathrm{m}$ and a distribution as follows: $10 \% \leq 0.7 \mu \mathrm{m}, 50 \% \leq 2.6 \mu \mathrm{m}$ and $90 \%$ $\leq 16.5 \mu \mathrm{m}$. These results demonstrate a shift in population from 10-20 microns to 1-10 microns. Furthermore, measuring the droplet size distribution 7 days later confirmed the presence of a stable emulsion. Although to the authors' knowledge, no work has been done to examine high pressure shock wave interaction with emulsions, ultrasonic approaches have been extensively studied e.g., [10]. Current thinking suggest that emulsification by ultrasound at least is governed by a localised cavitation zone [11]. These results are consistent with our shock studies. However, as noted above, a certain degree of 

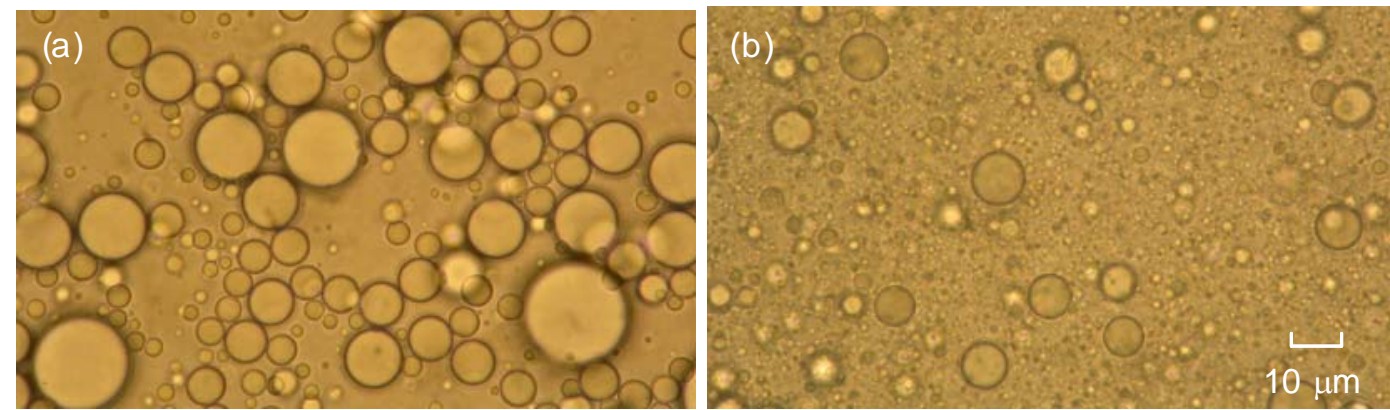

Figure 4. (a) Emulsion before treatment and (b) emulsion after the passage of the shock wave (with air gap).

emulsification has occurred where mostly cavitation was suppressed. Consequently, a different mechanism of break up may be at play and this demands explanation. One possibility is that the divergent wave front reflected from the deforming cover plate (see Fig 2) is inducing shear in the oil droplets. This may well be expected to cause a breakdown of the droplets however we are unable to verify this using the current setup.

\section{CONCLUSIONS}

Different bacterial suspensions and an emulsion have been subjected to shock loading where the magnitude of the shock wave in the stainless steel was measured to be $6.2 \mathrm{GPa}$ and $8.5 \mathrm{GPa}$ resulting in a shock pressure in the broth of up to c.a. 1.2 GPa. We have shown that when cavitation was both reduced and encouraged, the shock pressure had little effect on microorganisms such as $Z$. bailii, E. coli and E. faecalis. However, stable emulsification of a coarse oil and water emulsion did occur when cavitation was promoted. Considerably less emulsification occurred when cavitation was mostly suppressed.

Other researchers have noted significant bacterial kill with much lower pressures $[1,5]$. This work confirms that the mortality seen previously was not dependent on purely the magnitude of the pressure but probably on other factors present in those experiments such as degree of shock repetition and exposure to UV light.

\section{ACKNOWLEDGEMENTS}

Funding was provided by DEFRA under the LINK collaborative research programme (project
AFM 264). The authors thank Mr. Gary Cooper and Mr. Gareth Appleby-Thomas for providing valuable experimental support.

\section{REFERENCES}

1. Loske, A. M., et al., "Repeated application of shock waves as a possible method for food preservation", Shock Waves 9, 49, 1999.

2. Burchell, M. J., et al., "Survival of bacteria and spores under extreme shock pressures”, Mon. Not. R. Astron. Soc., 352, 1273, 2004.

3. Willis, M. J., et al., "Bugbuster—survivability of living bacteria upon shock compression”, Earth Planet. Sci. Lett., 247, 185, 2006.

4. Stöffler, D., et al., "Experimental evidence for the potential impact ejection of viable microorganisms from Mars and Mars-like planets”, Icarus 186, 585, 2007.

5. Zuckerman H., et al., "Inactivation of microorganisms using pulsed high underwater discharges”, Innovative Food Science and Emerging Technologies 3, 329, 2002.

6. Bourne, N.K., "A $50 \mathrm{~mm}$ bore gas gun for dynamic loading of materials and structures", Meas. Sci. Technol. 14, 273, 2003.

7. Caupin, F., and Herbert, E., "Cavitation in water: a review”, C. R. Physique, 7, 1000, 2006.

8. Mittal, N., et al., "Motility of Escherichia coli cells in clusters formed by chemotactic aggregation", PNAS 100, 13259, 2003.

9. Domingues, L., et al., "Applications of yeast flocculation in biotechnological processes", Biotechnol. Bioprocess. Eng., 5, 288, 2000.

10. Wood, R. W., and Loomis, A. L., "The physical and biological effects of high-frequency sound waves of great intensity”, Philos. Mag., 4, 417, 1927.

11. Cucheval, A. and Chow, R. C. Y., "A study on the emulsification of oil by power ultrasound", Ultrason. Sonochem., 15, 916, 2008. 